\title{
Coma Structures in Comet 73P/Schwassmann- Wachmann 3, Components B and C, Between January and May 2006
}

\author{
Jean-Baptiste Vincent • Hermann Böhnhardt • Ivano Bertini • \\ Luisa-Maria Lara $\cdot$ Michael Küppers $\cdot$ Rafael Rodrigo
}

Received: 12 December 2008/ Accepted: 19 September 2009/Published online: 25 October 2009

(C) The Author(s) 2009. This article is published with open access at Springerlink.com

\begin{abstract}
The Jupiter family comet 73P/Schwassmann-Wachmann 3 has been widely observed since 1995 after a nucleus break-up event produced at least five components labeled 73P-A to E. During the 2006 appearance, two of them (B and C) showed very strong coma activity. Our R-filter imaging of 73P-B \& C from 21 January to 25 May 2006 revealed the presence of fan-like structures in the comae of both components and evidence for further fragmentation events in component B. As of early April 2006, component C showed two jets emanating from the nucleus, with one continuously visible. Through a simulation of the orbital geometry we infer that the rotation axis of $73 \mathrm{P}-\mathrm{C}$ has an inclination of $20^{\circ}$ to the orbital plane and a longitude of $45^{\circ}$ at perihelion. The coma activity of component B was highly variable, displaying signatures of at least 3 fragmentation events. The coma was characterized by the continuous presence of a jet roughly in sunward direction, starting from the beginning of May. The first fragmentation event of component B may have happened between April 16 and April 26, leading to the presence of at least 6 fragments detected in images of May 2. The second one happened on or shortly before May 8 , the third one between May 18 and 24. For the rotation axis of 73P-B we infer an inclination of $5^{\circ}-15^{\circ}$ to the orbital plane and a longitude of $20^{\circ}-30^{\circ}$ at perihelion.
\end{abstract}

Keywords Schwassmann-Wachmann $3 \cdot 73 \mathrm{P} \cdot$ Comet · Coma structures · Morphology $\cdot$ Rotation axis

J.-B. Vincent $(\varangle) \cdot$ H. Böhnhardt

Max-Planck Institut für Sonnensystemforschung, Max-Planck-Str. 2,

37191 Katlenburg-Lindau, Germany

e-mail: vincent@mps.mpg.de

I. Bertini · L.-M. Lara · R. Rodrigo

Instituto de Astrofísica de Andalucía, CSIC, PO Box 3004, 18080 Granada, Spain

M. Küppers

European Space Astronomy Center (ESAC), PO Box 78,

28691 Villanueva de la Canada, Madrid, Spain 


\section{Introduction}

Discovered in 1930, the Jupiter Family comet 73P/Schwassmann-Wachmann 3 (73P) has been widely observed after it broke up in at least five components during its perihelion passage in autumn 1995 (Böhnhardt et al. 1995). During the 2001 perihelion passage, these components appeared as individual comets and no particular events were found (Böhnhardt et al. 2002; Böhnhardt 2004). In 2006, the 5.34 years periodic comet came very close to Earth (minimum geocentric distance of 0.079 AU on 12 May for component $\mathrm{C}$, and 0.067 AU on 14 May for component B), just before the perihelion passage in early June 2006. This orbital configuration was a good opportunity to investigate the morphology of the coma of the two main components 73P-B \& 73P-C, constrain the rotation axis, and detect new fragmentation events.

We used a sequence of 21 optical broadband images acquired with the CAFOS instrument at the 2.2-m telescope at Calar Alto Observatory (CSIC-MPG) as described in Bertini et al. (2009). These images depict the evolution of the coma between 21 January and 25 May 2006 for component C, and between 8 February and 24 May 2006 for component $\mathrm{B}$. In order to determine whether there were some morphological structures in the comae of the 73P/Schwassmann-Wachmann 3 components, we enhanced calibrated $\mathrm{R}$ filter images using two different methods: 1- Adaptive Laplace filtering as described in Böhnhardt et al. (1994) and references therein; 2- Radial normalization for verification of the features found through the Laplace filtering. An independent verification of possible coma structures was also done by checking for anisotropies in the isophotes of the calibrated images.

\section{Coma Structures of Component $\mathrm{C}$}

\subsection{Global Description}

From 21 January to 4 March 2006, component $C$ was active but did not show any specific structure in the coma morphology, except for the dust tail that is always present roughly in antisolar direction. On 4 March (1.57 AU from the Sun), the coma appeared to be elongated in the sunward direction at position angle $\mathrm{PA}=60^{\circ}$ (measured counterclockwise from the North). This structure evolved during the month and on 5 April it turned out to be a fan-like coma pattern (see Fig. 1). Two jet-like features (A and B in Fig. 1) with a typical extension of $\sim 7000 \mathrm{~km}$ (projected distance from the nucleus) could be identified at PA = $16^{\circ}-53^{\circ}$. In April, the pattern A was continuously present in all our images in PA range $7^{\circ}-$ $25^{\circ}$. Contrary to structure A, pattern B showed a variable appearance. On 13 April it appeared to be disconnected from the maximum intensity peak in our image (i.e. the location of the nucleus) and was not seen in the coma on 16 April, but appeared again with a strong intensity on 26 April. On 8, 9, 10 May, both structures A \& B were visible at the same time, with similar characteristics (extension, orientation, curvature). On 10 and 11 May, they faded away; however we could still notice a small elongation of the coma in the direction of the former structures. Pattern A appeared again on 12 May with a very strong intensity, while pattern B showed up only the day after. The two jets remained in the coma until 14 May when they faded away again. On 16 May we could see only a small elongation of the coma in the direction of structure $\mathrm{A}$ at $\mathrm{PA}=45^{\circ}$. Jet A appeared again on 18 May, extending to $\sim 2700 \mathrm{~km}$ on 24 May, but it is not present anymore in our last image (25 May). Table 1 gives more detailed information about the jets. In summary, although 


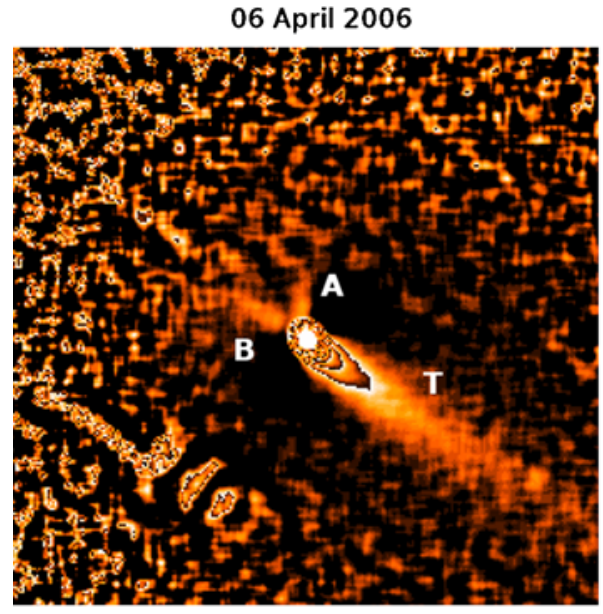

12 May 2006

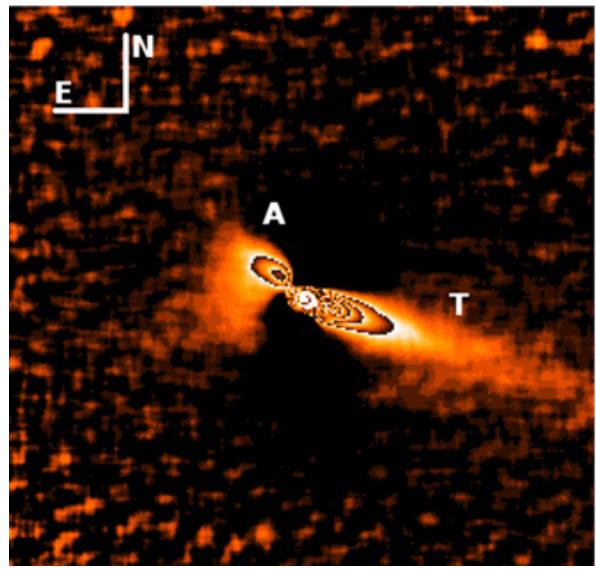

16 April 2006

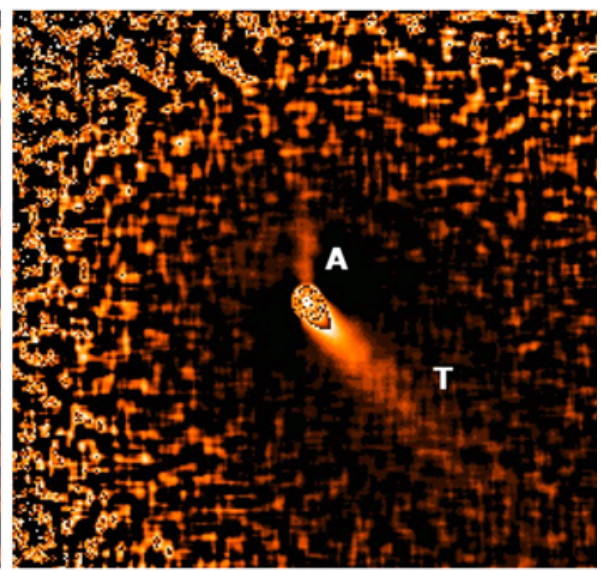

14 May 2006

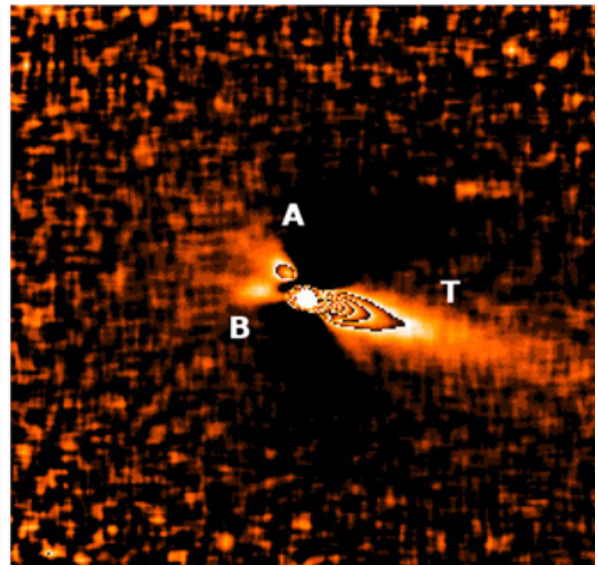

Fig. 1 Images of comet 73P/Schwassmann-Wachmann 3 component $C$, enhanced by Laplace filtering, showing the two coma structures (A and B) and the dust tail (T). The observing dates are listed at the top of the four panels. The field of view is $135^{\prime \prime} \times 135^{\prime \prime}$. The color scale represents the location of a morphological structure and not the brightness intensity as this information is lost during the Laplace filtering

variable in intensity, feature A is present from the beginning of April 2006 to the end of our observations on late May 2006. The second pattern B cannot be seen on all the exposures; it seems to appear and disappear with a period of about 2-3 days.

\subsection{Interpretation}

As the contribution of light from gas and ion emissions is small in the $\mathrm{R}$ band, we attribute the observed structures (tail T, patterns A \& B) to dust reflected sunlight. Following the approach of Sekanina (1987), we consider patterns A \& B as part of a coma fan structure produced by active dust-emitting sources on the rotating nucleus. Considering a singlesource on a rotating spherical nucleus, we can interpret the observed pattern as an emission cone viewed side on, with the borderlines forming the straight features A and B in the enhanced images. Therefore, the projected position of the rotation axis falls in between the 
Table 1 Geometry of the Sun and coma structures in 73P-C images

\begin{tabular}{|c|c|c|c|c|c|}
\hline \multirow[t]{2}{*}{ Date } & \multirow[t]{2}{*}{ Sun } & \multicolumn{2}{|c|}{ Position angle } & \multicolumn{2}{|l|}{ Extension } \\
\hline & & Struct. A & Struct. B & Struct. A & Struct. B \\
\hline 2006-Jan-21.14 & 107.7 & ND & ND & - & - \\
\hline 2006-Feb-07.25 & 101.6 & $\mathrm{ND}$ & ND & - & - \\
\hline 2006-Mar-04.07 & 86.1 & 60 & $\mathrm{ND}$ & 8700 & - \\
\hline 2006-Mar-31.06 & 55.3 & 40 & ND & 4950 & - \\
\hline 2006-Apr-05.99 & 47.7 & 16 & 53 & 6150 & 5600 \\
\hline 2006-Apr-11.12 & 41.9 & 22 & 68 & 5050 & 4500 \\
\hline 2006-Apr-13.18 & 39.9 & 24 & 66 & 6950 & 5350 \\
\hline 2006-Apr-16.01 & 37.5 & 7 & ND & 3600 & - \\
\hline 2006-Apr-26.06 & 35.0 & 25 & 69 & 2650 & 2600 \\
\hline 2006-May-02.06 & 41.2 & 26 & $\mathrm{ND}$ & 2750 & - \\
\hline 2006-Мay-08.18 & 57.7 & 16 & 65 & 1350 & 1350 \\
\hline 2006-Мay-09.17 & 61.1 & 35 & 95 & 1850 & 1800 \\
\hline 2006-May-10.15 & 64.3 & 64 & 71 & 500 & 500 \\
\hline 2006-May-11.07 & 67.2 & 63 & 87 & 1000 & 1000 \\
\hline 2006-May-12.15 & 70.0 & 48 & ND & 1450 & - \\
\hline 2006-May-13.17 & 72.0 & 33 & 85 & 1400 & 1700 \\
\hline 2006-Мay-14.15 & 73.3 & 31 & 87 & 1100 & 950 \\
\hline 2006-Мay-16.15 & 74.2 & 45 & ND & 850 & - \\
\hline 2006-May-18.15 & 73.3 & 47 & ND & 1350 & - \\
\hline 2006-May-24.17 & 66.8 & 65 & 65 & 2800 & 2800 \\
\hline 2006-May-25.15 & 65.8 & 43 & ND & 2100 & 2100 \\
\hline
\end{tabular}

This table gives the near nucleus position angle of the two structures A and B described in the text, together with the position angle of the Sun. Angles are measured counterclockwise from the North in the sky plane of the observer (North $=0^{\circ}$, East $=90^{\circ}$ ) and refer to the estimated central lines of the features observed. An uncertainty of about $5^{\circ}$ should be considered. The extension of the features projected in the sky is given in kilometers

$N D$ not detected

angle formed by the two patterns and the aperture angle of the fan is directly related to the distance between the source and the pole of the rotating nucleus. Table 1 gives the evolution of the mean position angle of the fan formed by the two structure. The rotation axis and the temporal evolution of its projected position on the sky plane as obtained in a simulation of the comet orbit must be compatible with these measurements. Using a trial and error approach, varying the rotation axis systematically over the $4 \pi$ sphere of the nucleus, we could constrain the orientation of the spin axis. A refined simulation gave the best match between predicted and measured position angle of the projected cone axis for a rotation axis orientation with an inclination of $20^{\circ}-25^{\circ}$ to the orbital plane and a longitude of $40^{\circ}-45^{\circ}$ at perihelion. Inclination is defined as the angle between the spin axis and the orbital plane (i.e. $90^{\circ}$-obliquity), and zero longitude is along the extended sun-comet vector at perihelion. The best fit of this solution to the measurements (error minimized with a least square method) is presented in Fig. 2. This solution assumes a fixed rotation axis and does not consider precession. 


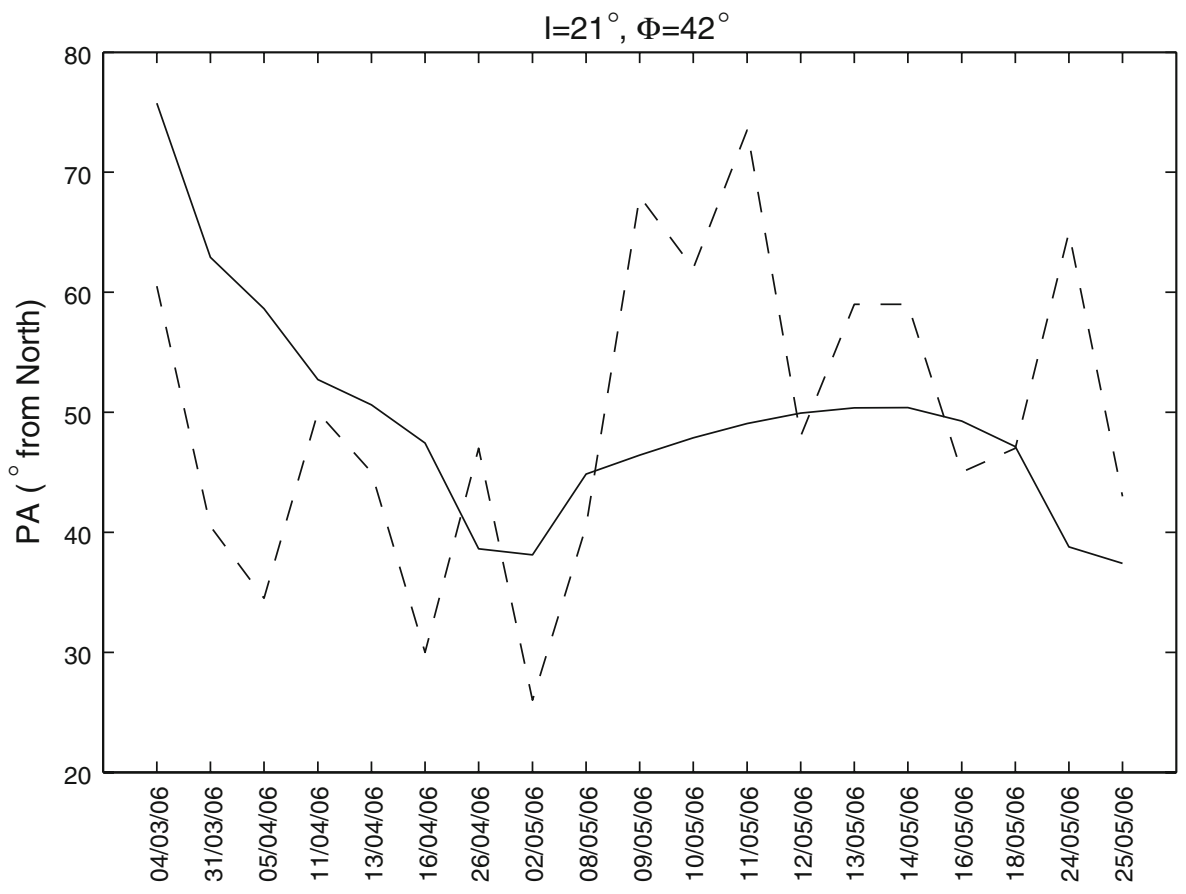

Fig. 2 Best fit of the position angle of the projected rotation axis position of 73P-C for our observation dates. The dashed line gives the mean position angles of the two structures A \& B described in the text. The solid line is the projected position angle of the best fit rotation axis

\section{Coma Structures of Component B}

The overall evolution of the coma structure for component B is quite different from the component $\mathrm{C}$ one and shows more variability in time and amplitude. There are also clear indications of nucleus fragmentations.

\subsection{Global Description}

From 8 February to 5 April 2006, the images of component B displayed only an elliptical but featureless coma together with the dust tail. From 11 to 16 April, the coma appeared more and more elongated along the nucleus-tail direction. On 26 April, the isophotes were strongly distorted and the Laplace filtered images showed the presence of several fragments on the tailward side of component B. They remained for several days in the coma and their number increased to at least 6 on 2 May. These fragments were not found anymore in the exposure on 8 May. On 2 May, we also observed the presence of a sunward structure (identified by an A in Fig. 3) which remained visible until 13 May. On 8 May, we noticed the appearance of arclet structures (identified as W1 \& W2 in Fig. 3, second row), with orientation roughly perpendicular to the tail direction. The projected extension of these features varied from $\sim 800 \mathrm{~km}$ to $\sim 5000 \mathrm{~km}$ over a single day (from 8 May morning to 9 May morning). In the next observing night (9 May), the two arclets W1 \& W2 appeared to be disconnected from the inner part of the coma (nucleus location). They were not present anymore on 10 May. On 13, 14, and 16 May, we observed again some small fragments in the tail region of component B. 


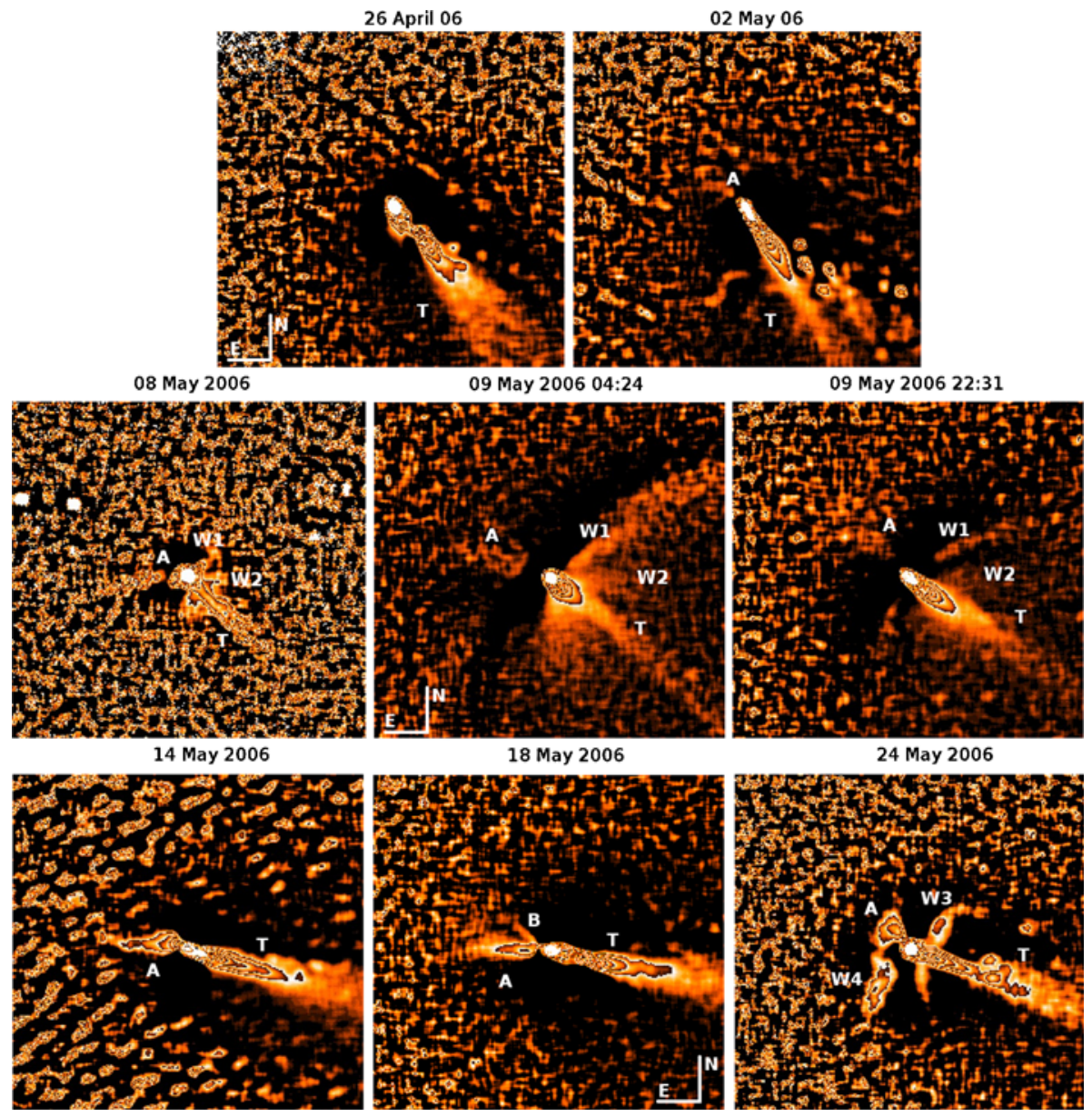

Fig. 3 Images of comet 73P/Schwassmann-Wachmann 3 component B, enhanced by Laplace filtering, showing various morphological structures. First row displays small fragments in the tail region of the coma. Second row shows arc structures (W1 and W2), a sunward fan (A), and the dust tail (T). Last row shows high variability of the coma structures in the second half of May 2006. Orientation and dates given in the figure, field of view $135^{\prime \prime} \times 135^{\prime \prime}$

We also noticed a very strong increase of activity in the sunward direction. Structure A extends up to $1950 \mathrm{~km}$ on 14 May. On 18 May, a second structure (B in Fig. 3, third row) is found in the sunward coma hemisphere at an angle of $-45^{\circ}$ with respect to pattern A. This feature disappeared (or merged with the other one) in our last image on 24 May. The latter showed also the presence of two fragments, along with the appearance of new arclets (W3 \& W4) similar to those observed on 9 May. Tables 2, 3, \& 4 give more detailed information about the structures in the coma of 73P-B.

\subsection{Interpretation}

In our images of component B we found evidences for several fragmentation events. On 26 April we note a strong deformation of the isophotes along with the presence of a small 
Table 2 Geometry of the Sun and coma fans in 73P-B images, together with the extension of the structures

\begin{tabular}{|c|c|c|c|c|c|}
\hline \multirow[t]{2}{*}{ Date } & \multirow[t]{2}{*}{ Sun } & \multicolumn{2}{|c|}{ Position angle } & \multicolumn{2}{|l|}{ Extension } \\
\hline & & Struct. A & Struct. B & Struct. A & Struct. B \\
\hline 2006-Мау-02.09 & 29.0 & 44 & - & 2000 & - \\
\hline 2006-Мay-08.19 & 44.4 & 95 & - & 2200 & - \\
\hline 2006-Мау-09.18 & 48.7 & 65 & - & 2600 & - \\
\hline 2006-Мay-09.94 & 52.2 & 45 & - & 2500 & - \\
\hline 2006-May-10.96 & 57.2 & 41 & - & 1700 & - \\
\hline 2006-Мay-13.01 & 66.8 & 48 & - & 1600 & - \\
\hline 2006-Мay-14.04 & 70.7 & 91 & - & 1950 & - \\
\hline 2006-Мay-16.12 & 75.4 & 61 & - & 2000 & - \\
\hline 2006-May-18.14 & 75.9 & 92 & 45 & 2550 & 1600 \\
\hline 2006-Мау-24.15 & 68.2 & 46 & - & 1250 & - \\
\hline
\end{tabular}

For an explanation of the table columns, see Table 1

Table 3 Geometry of the arclets in 73P-B

\begin{tabular}{lllll}
\hline Date & Id. & Orientation & Curvature & Extension \\
\hline 2006-May-08.19 & W1-E/W1-W & $125 / 315$ & S/S & $500 / 1000$ \\
& W2-E/W2-W & $160 / 270$ & CC/C & $800 / 800$ \\
$2006-$ May-09.19 & W1-E/W1-W & $125 / 300$ & CC/C & $2600 / 4900$ \\
& W2-E/W2-W & $135 / 265$ & CC/C & $2300 / 2600$ \\
2006-May-09.94 & W1-E/W1-W & $161 / 296$ & CC/C & $3300 / 3600$ \\
$2006-M a y-24.15$ & W2-E/W2-W & $192 / 273$ & CC/C & $1550 / 1950$ \\
& W3-E/W3-W & $180 / 335$ & CC/C & $1400 / 1800$ \\
& W4-E/W4-W & $35 /-$ & CC/- & $2450 /-$ \\
\hline
\end{tabular}

This table gives the near nucleus position angle of coma arclets described in the text. The angles are measured counterclockwise from the North in the sky plane of observer (North $=0^{\circ}$, East $=90^{\circ}$ ) An uncertainty of about $5^{\circ}$ should be considered. The extension of the features projected in the sky is given in kilometers. Our values differ slightly from the ones measured by Bonev et al. (2008) which might be due to a better signal to noise ratio in their data set or activity rapidly evolving with time. Arclets identifications are the same as in Fig. 3. The label $-\mathrm{E}$ or $-\mathrm{W}$ indicates eastern or western wing of the arclet

$S$ straight, $C$ clockwise, $C C$ counterclockwise

fragment in the tail direction. As the previous image (taken on 16 April) did not show anything but the dust tail, we believe that a fragmentation event occured in between these two dates, leading to the creation of the small pieces observed on 26 April and 2 May (see Fig. 3, first row). This interpretation fits with amateur observations reporting a significant outburst on the component B during the early hours of 24 April, which caused a significant increase in the overall brightness until 27 April (see http://www.kometen.fg-vds.de/koj_2006/73p/ 73 peaus.htm). On 2 May, the brightest of the at least six fragments was only about $300 \mathrm{~km}$ (projected distance) from the nucleus and it was almost as bright as the main coma peak of component B. The position of these fragments is given in Table 4. Evidence of a second fragmentation event comes from the presence of arclets in the images from 8 to 10 May (Fig. 3, second row). As described in Böhnhardt (2004), these arclets are signature of nucleus fragmentation, and should mainly consist of gas. Their occurence lasted about 2 days and was 
Table 4 Position of the small pieces observed in the tail direction of component B on 26 April and 2 May 2006

\begin{tabular}{llr}
\hline Date & x/y & Dist. \\
\hline 2006-April-26.08 & $-16.2 /-23.1$ & 3366 \\
2006-May-02.09 & $-14.9 /-20.9$ & 2271 \\
& $-22.4 /-22.7$ & 2792 \\
& $-24.7 /-32.2$ & 3549 \\
& $-32.5 /-30.1$ & 3880 \\
& $-32.9 /-59.8$ & 6057 \\
\hline
\end{tabular}

$\mathrm{X} / \mathrm{Y}$ coordinates (in arcsec) of the small fragments appearing in the tail direction on 26 April and 02 May 2006, as described in the text. Positive $\mathrm{X}$-axis points toward the North and positive $\mathrm{Y}$-axis towards the East. The distance (in $\mathrm{km}$ ) is the projected distance between each fragment and the central peak of brightness in the coma of 73P-B. Ucertainty on the position is about $0.15^{\prime \prime}$ (corresponds to $18 \mathrm{~km}$ on 26 April and $13 \mathrm{~km}$ on 2 May). It remains unclear if the secondary fragment seen on 26 April is identical to one of the 6 fragments seen on 2 May 2006

also recorded by observers at the National Astronomical Observatory (NAO) Rozhen, Bulgaria (Bonev et al. 2008). Unfortunately, we do not have images close enough in time to the fragmentation event that could illustrate the growth of the arclets. However, as the formation of these wings is mostly driven by gas expansion, with typical velocities of a few hundred meters per second we can assume that the fragmentation event should have occured on 8 May, a few hours before our observation. We note that on our next images $(10,13,14,16$ May) we did not observe evidence of fragments that might be associated with the event described above: either the pieces were quickly destroyed, or they were too small to be detected.

We also noticed in these images an increase in intensity of the sunward fan, along with a strong distortion of the coma isophotes. On one side it can be due to a more favorable illumination of the active region, but as it happened immediately after the fragmentation event, both phenomena might very well be related. For instance the fragmentation event could have lead to the exposure of fresh material at the surface of the nucleus, thus creating a new active area with increased emission of gas and dust. This scenario is supported by the presence of a second structure on 18 May, seemingly coming from the same region (identified as B in Fig. 3, third row). This pattern could be the signature of a second active source at the surface of the nucleus. In our last exposure of 24 May, only a single structure is visible which might be either feature $\mathrm{A}$ or the result of a merging of feature $\mathrm{A}+$ feature $\mathrm{B}$ in case of two different active regions. As described before, this exposure showed also two arclets that might be signatures of yet an earlier fragmentation event.

As for 73P-C, we tried to constrain the attitude of the rotation axis for component B, using the measured position angles of the sunward coma fan. The results are not as accurate as for component $\mathrm{C}$ because this fan is present only in 10 images, covering a time span of 22 days, which implies less geometrical evolution of the projected axis (whereas we could follow the evolution during 3 months for 73P-C). Nevertheless we obtain a best match for the rotation axis with an inclination $5^{\circ}-15^{\circ}$ to the orbital plane and a longitude $20^{\circ}-30^{\circ}$ at perihelion.

\section{Conclusions}

From the analysis of the coma structures in comet $73 \mathrm{P}$ before perihelion in 2006 , we found that the two main components ( $\mathrm{C}$ and $\mathrm{B}$ ) behave differently during most of the time span of our observations: 
- As of early April 2006 73P-C shows two jets emanating from the nucleus. Although variable in intensity, the first one is always present while the second is appearing and disappearing with a period of two to three days. Through Monte Carlo dust coma modeling we inferred that the best possible rotation axis of SW3-C has a low inclination of $20^{\circ}-25^{\circ}$ to the orbital plane and a longitude of $40^{\circ}-45^{\circ}$ at perihelion (zero in longitude is the Sun-comet extended vector).

- 73P-B displayed a higher variability in terms of activity, showing also several fragmentation events. Its coma is characterized by the continuous presence of a jet roughly in sunward direction, starting from the beginning of May, and by several arclets structures connected to fragmentation events of the nucleus. From the morphological analysis of the coma structures we detect three fragmentation events. The first one happened between April 16 and April 26, leading to the presence of several fragments on May 2. The second can be happened on May 8, the third one before May 24. From the Monte Carlo modeling of the dust we infer a rotation axis of $73 \mathrm{P}-\mathrm{B}$ with again a low inclination of $5^{\circ}-15^{\circ}$ to the orbital plane and a longitude of $20^{\circ}-30^{\circ}$ at perihelion.

Open Access This article is distributed under the terms of the Creative Commons Attribution Noncommercial License which permits any noncommercial use, distribution, and reproduction in any medium, provided the original author(s) and source are credited.

\section{References}

I. Bertini, L.M. Lara, J.B. Vincent, H. Boehnhardt, M. Küppers, R. Rodrigo, Activity evolution, outbursts, and splitting events of comet 73P/Schwassmann-Wachmann 3. A \& A 496, 235-247 (2009)

H. Böhnhardt, K. Birkle, Time variable coma structures in comet P/Swift-Tuttle. A \& A 107, 101-120 (1994)

H. Böhnhardt, S. Holdstock, O. Hainaut, G.P. Tozzi, S. Benetti, J. Licandro, 73p/Schwassmann-Wachmann 3-One Orbit after Break-Up: search for fragments. Earth Moon Planets 90, 131-139 (2002)

H. Böhnhardt, Split comets. Comets II pp. 301-316 (2004)

H. Böhnhardt, H.U. Kaufl, R. Keen, P. Camilleri, J. Carvajal, A. Hale, Comet 73P/Schwassmann-Wachmann 3. IAUC 6274 (1995)

T. Bonev, H. Böhnhardt, G. Borisov, Broadband imaging and narrowband polarimetry of comet $73 \mathrm{P} /$ Schwassmann-Wachmann 3, components B and C, on 3, 4, 8, and 9 May 2006. A \& A (2008)

Z. Sekanina, Anisotropic emission from comets: fans versus jets. i. concept and modelling. ESA SP-278 pp. 315-322 (1987) 